\title{
A Class of Improved Wake Interaction Model for the Coordinated Control of Wind Farms
}

\author{
Lin Pan, Holger Voos, Yumei Li \\ Interdisciplinary Centre for Security, \\ Reliability and Trust, \\ University of Luxembourg, Luxembourg \\ Email: lin.pan@uni.lu,holger.voos@uni.lu,yumei.li@uni.lu \\ Yuhua Xu \\ School of Finance, Nanjing Audit University, \\ Jiangsu 211815, China \\ Email: yuhuaxu2004@163.com
}

\author{
Mohamed Darouach \\ Research Center for Automatic Control of Nancy \\ (CRAN UMR, 7039, CNRS), \\ University of Lorraine, France \\ Email: mohamed.darouach@univ-lorraine.fr \\ Lin Pan, Zhansheng Li \\ School of Electric \& Electronic Engineering, \\ Wuhan Polytechnic University, Wuhan, China \\ Email: linpandr@gmail.com,704849831@qq.com
}

\begin{abstract}
This paper is focused on the Wind Energy (WE) systems and Wind Farms (WFs) optimization in Luxembourg. It describes the overview of the wind resources in all the WFs and presents a class of Improved Wake Interaction Model (IWIM) for Coordination and Optimization Control (CnOC) of WFs. Based on Wind Power (WP) assessment of WFs, the statistical method is used to model the distribution of Wind Speed (WS) and Wind Direction (WD). Some simulation figures about the Wind Rose (WR) and WF optimization demonstrate the description and assessment of WP in detail. These assessments are expected to enhance the effectiveness of exploitation and utilization of WP in WFs of Luxembourg.
\end{abstract}

Index Terms-Wind Energy, Wind Farms, Wind Turbine, Wind Rose, Wind Power, Tip Speed Ratio.

\section{Introduction}

It is well known that WE is widely recognized to be one of the most cost-efficient renewable sources of energy. With the increase of global wind-generation capacity in the last five years, WE has also become the fastest-growing electrical energy in the world. In the processes of Wind Energy (WE) utilization, Wind Power (WP) assessment is a critical step. In order to enhance the effective utilization of WE, the best way is to utilize existing WFs through improving control techniques and algorithms. At present, WE systems are being inclined to develop into large-scale distributed and coordination systems where there are even more than eighty individual Wind Turbines (WTs) in operation. In contrast to the conventional power plants, e.g. nuclear power, thermal power, hydropower, etc. [1], [2], [3], [4], [5], [6], [7], [8], these wind devices and equipments are expected to operate and provide high quality power (Such as: Safe, Stable, Controllable and Predictable (SSCP)) at the lowest possible cost.
Recently, the research and development of WE harvesting systems were focused on optimizing different aspects of WTs in order to improve its Cost of Energy (CoE). Furthermore, some challenging problems, e.g. the electric energy product increment and the cost reduction emerged from the rapid growth of the WE industry. The WTs location optimization is an effective way to reduce the wake effect of WTs and to increase the total power output of the WF. Increase performance of the control system by optimizing WTs controller [1], [2] is one of the most important ways to enhance CoE of WTs. WTs are often installed in wind parks or wind farms on a large scale as so to reduce costs in scale. Turbines in WFs can be located along a single line, in multiple lines, in grids, in clusters or in configurations based on geographical features, prevailing WD, access requirements, environmental effects, safety, prior and future land use including ranch-land and farmland, and visual impact [3], [4].

Recent years, WE is developing rapidly in Luxembourg. In view of Luxembourg locating in the western central area of European, and there are abundant wind resources to tap into Luxembourg. There are currently more than 16 WFs established in the different places of Luxembourg. From control perspective, the farm level control is the most challenging task. The complexity of large scale farms is handled by a hierarchical approach, separating the control into the following levels, for example, the WF level and control on the single WT level [7]. The operator level controller serves the demands from the network operator that gives a set point for active and reactive power for the whole farm combined with one of several operational modes, e.g. maximum energy production, rate limiting, balancing, frequency control, voltages control, or delta control. On the lowest control level, the single WT level, the respective set points are achieved via coordinated control of a wind turbine array for power maximization which is described in [7]. Meanwhile, the most challenging task is the farm level 
control. Inspired by the above works and discussions, we argue that the key and core research contents are to operate WFs with the efficient methods, to improve WP, power quality, and energy capture of the overall WTs. Moreover, for reducing complex structural electricity loading, advanced and hybrid control measures have to be applied on the WFs. However, there is still lack of effective control method of WFs which requires further research and development. All factors mentioned above motivate our research in this field.

Due to the complex interactions among the WTs, the challenging problem of WFs is the fact that the pure control on the single-WT is not completely sufficient. Firstly, all WTs in a farm are connected to the same Electrical Grid (EG) and each individual WT therefore has an influence on the quality or output of the generated electrical power. Secondly, there are also aerodynamic interdependencies among the WTs because each WT influences the airstream that flows through the WF. In addition, also these flows influence the wind conditions and hereby the power generation as well as the electricity structural load on other WTs. Furthermore, aerodynamic and electrical interaction among WTs in a WF also result in the losses of energy-capture, which have to be recovered by a suitable control measures. Therefore, the overall control strategy of WFs is currently a very important research topic and also the main objective which goes far beyond the control method of a single WT.

The main contribution of this paper are as follows. Firstly, an Improved Actuator Disk Model (IADM) for WFs is proposed for the firs time. According to the conservation of mass and momentum, some important equations with their derivations and proofs give the main variables and features of the IADM. Secondly, an Improved Jensen Wake Model (IJWM) with it characteristic for WFs are presented and studied in detail. Further, wind power generation system is studied, and wind rose and wind weibull probability density distribution are elaborated and discussed that all focused on WFs of Luxembourg. Finally, the problem of WTs location optimization is discussed. Some simulation figures are provided to prove their effectiveness and correctness in the paper.

The paper is organized as follows: In section 2, IADM is described and introduced. In section 3, an IJWM for WF are obtained and discussedThe wind rose and wind Weibull probability density distribution are presented in section 4 . Finally, the conclusion is summarized in section 5.

\section{An Improved Actuator Disk Model (IADM) for WFs}

Fig. 1 describes a Improved Actuator Disk Model (IADM), which theory is described by a mathematical model with an ideal state. The model was first proposed or originated by W.J.M. Rankine in 1865, Alfred George Greenhill in 1888 and R.E. Froude in 1889 [10]. In this section, we will discuss an IADM.

Let's assume that the wind is the ideal fluid, and has no fraction, viscosity and turbulence. The wind turbine is seen

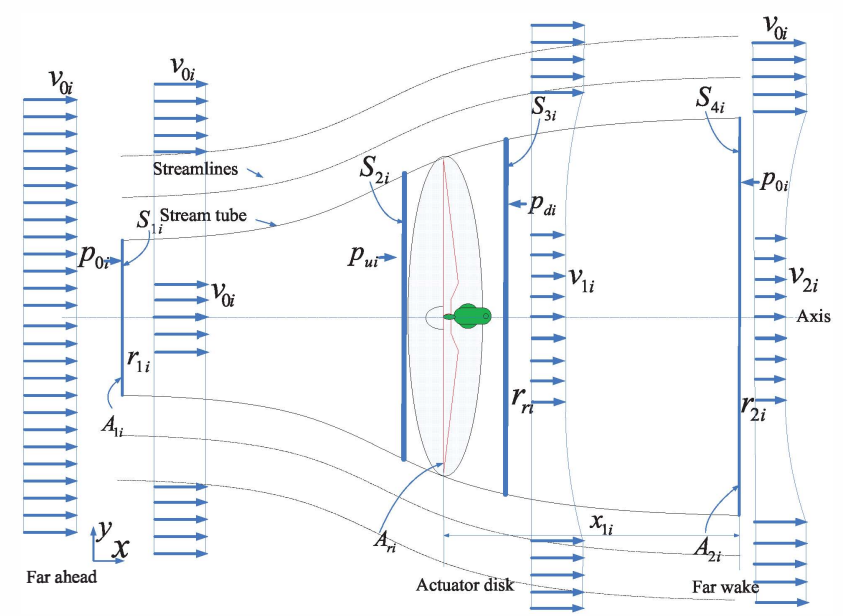

Figure 1. The portrait of actuator disk model with stream tube [9].

as a solid disk from which the wind can go through. The stream tubes are shown in Fig. 1. A stream tube is the region which lateral boundary is dense impermeable by the wind. A cross-section of a tube is defined to the axis of the tube as shown in this figure. There are four cross-sections are $S_{1 i}, S_{2 i}, S_{3 i}$ and $S_{4 i}$ which are shown in Fig. 1, respectively.

In front of the actuator disk is the cross-section $S_{2}$, and $S_{3}$ is behind it immediately. The wind speed on $S_{1 i}, S_{2 i}, S_{3 i}$ and $S_{4 i}$ are assumed to $v_{1 i}, v_{2 i}, v_{3 i}$ and $v_{4 i}$ respectively. The ambient pressures on upside and downside of the actuator disk are $p_{u i}$ and $p_{d i}$. The entrance rotor radius is $r_{1 i}$, and the actuator disk rotor radius is $r_{r i}$. The downstream rotor radius is $r_{2 i}$ with air pressure $p_{0 i}$. The second half of region is called the near wake region, and the length of the near wake region is defined by $x_{1 i}$.

Using the conservation of mass, we conclude that:

$$
\begin{aligned}
\sum_{i=1}^{n} \rho A_{1 i} v_{0 i} & =\sum_{i=1}^{n} \rho A_{r i} v_{1 i} \\
& =\sum_{i=1}^{n} \rho A_{2 i} v_{2 i}=\sum_{i=1}^{n} \dot{M}_{i}, \quad n=1,2, \cdots, N .
\end{aligned}
$$

where $\rho$ is the air density, the cross-sectional areas of $S_{1 i}$, the actuator disk and $S_{4 i}$ are $A_{1 i}, A_{r i}$ and $A_{2 i}$ respectively. $N$ is the number of the WTs in the WF. The rate of flow of mass in stream tube is defined by $\dot{M}_{i}$. Given the radius of the actuator disk and $A_{2 i}$, we can get area of them: $A_{r i}=\pi r_{r i}^{2}$ and $A_{2 i}=\pi r_{2 i}^{2}$. The Axial Induction Factor (AIF) $\sum_{i=1}^{n} a_{i}$ can be define by:

$$
\sum_{i=1}^{n} a_{i}=\sum_{i=1}^{n}\left(\frac{v_{0 i}-v_{1 i}}{v_{0 i}}\right), \quad n=1,2, \cdots, N .
$$

The AIF $\sum_{i=1}^{n} a_{i}$ indicate the influence of the wind turbines on their surroundings. and then,

$$
\sum_{i=1}^{n} v_{1 i}=\sum_{i=1}^{n}\left(1-a_{i}\right) v_{0 i}, \quad n=1,2, \cdots, N
$$


The wind will give the thrust force $\sum_{i=1}^{n} T_{i}$ to the actuator disk and push them work. The direction of the thrust force and the wind velocity are same. Here $\sum_{i=1}^{n} T_{i}$ is different to the torque. $A_{1 i}$ and $A_{2 i}$ the outsides of the stream tube, although the cross-sectional areas of $S_{1}$ and $S_{4}$ are different, the air pressure on both of them are the same. If we only consider the ideal state, the net force on the air fluid in stream tube is equal to $T$. According to the momentum theory, we can obtain the following results:

$$
\sum_{i=1}^{n} T_{i}=\sum_{i=1}^{n}\left(\dot{M}_{i} v_{0 i}-\dot{M}_{i} v_{2 i}\right), \quad n=1,2, \cdots, N
$$

In the ideal state, Bernoulli's principle is applicable to the left and right parts of stream tube, we obtain the following two equations:

$$
\begin{aligned}
& \sum_{i=1}^{n}\left(p_{0 i}+\frac{1}{2} \rho v_{0 i}^{2}+\rho g h_{1 i}\right) \\
& =\sum_{i=1}^{n}\left(p_{u i}+\frac{1}{2} \rho v_{1 i}^{2}+\rho g h_{1 i}\right)=\text { Const }, \quad n=1,2, \cdots, N
\end{aligned}
$$

Herein, $g=9.8 \mathrm{~m} / \mathrm{s}^{2}$ is the acceleration of gravity on Earth.

$$
\begin{aligned}
& \sum_{i=1}^{n}\left(p_{d i}+\frac{1}{2} \rho v_{1 i}^{2}+\rho g h_{2 i}\right) \\
& =\sum_{i=1}^{n}\left(p_{0 i}+\frac{1}{2} \rho v_{2 i}^{2}+\rho g h_{2 i}\right)=\text { Const }, \quad n=1,2, \cdots, N .
\end{aligned}
$$

$h_{1 i}$ and $h_{2 i}$ are the heights of the fluid (from a reference point count). According to the equations (5) and (6), we get

$$
\sum_{i=1}^{n}\left(p_{u i}-p_{d i}\right)=\sum_{i=1}^{n}\left[\frac{1}{2} \rho\left(v_{0 i}^{2}-v_{2 i}^{2}\right)\right], \quad n=1,2, \cdots, N .
$$

Since the thrust force has the following equation:

$$
\sum_{i=1}^{n} T_{i}=\sum_{i=1}^{n}\left[\left(p_{u i}-p_{d i}\right) A_{r i}\right], \quad n=1,2, \cdots, N,
$$

we have

$$
\sum_{i=1}^{n} T_{i}=\sum_{i=1}^{n}\left[\frac{1}{2} \rho\left(v_{0 i}^{2}-v_{2 i}^{2}\right) A_{r i}\right], \quad n=1,2, \cdots, N .
$$

Actually, when $v_{2 i}$ is equal to 0 , the maximum $T_{\text {imax }}$ is achieved. So the theoretical $T_{\text {imax }}$ is defined by

$$
\sum_{i=1}^{n} T_{\text {imax }}=\sum_{i=1}^{n} \frac{1}{2} \rho v_{0 i}^{2} A_{r i}, \quad n=1,2, \cdots, N
$$

Substituting (1) and (8) into (4), and simplifying the resulting equation, we obtain

$$
\sum_{i=1}^{n} v_{1 i}=\sum_{i=1}^{n}\left[\frac{1}{2}\left(v_{0 i}+v_{2 i}\right)\right], \quad n=1,2, \cdots, N
$$

Substituting (11) into (3), $v_{2 i}$ is got as follows,

$$
\sum_{i=1}^{n} v_{2 i}=\sum_{i=1}^{n}\left[\left(1-2 a_{i}\right) v_{0 i}\right], \quad n=1,2, \cdots, N
$$

The thrust coefficient $C_{T i}$ is defined as

$$
\begin{aligned}
\sum_{i=1}^{n} C_{T i} & =\sum_{i=1}^{n} \frac{T_{\text {ThrustForcei }}}{T_{\text {DynamicForcei }}} \\
& =\sum_{i=1}^{n} \frac{T_{i}}{T_{\text {imax }}} \\
& =\sum_{i=1}^{n}\left[\frac{\frac{1}{2} \rho\left(v_{0 i}^{2}-v_{2 i}^{2}\right) A_{r i}}{\frac{1}{2} \rho v_{0 i}^{2} A_{r i}}\right] \\
& =\sum_{i=1}^{n}\left[\frac{2 \rho v_{0 i}^{2} A_{r i} a_{i}\left(1-a_{i}\right)}{\frac{1}{2} \rho v_{0 i}^{2} A_{r i}}\right], \quad n=1,2, \cdots, N .
\end{aligned}
$$

Substituting (12) into (13) and simplifying it, $C_{T i}$ is got:

$$
\sum_{i=1}^{n} C_{T i}=\sum_{i=1}^{n}\left[4 a_{i}\left(1-a_{i}\right)\right], \quad n=1,2, \cdots, N
$$

And other expressions about $C_{T i}$ are as follows:

$$
\begin{aligned}
& \sum_{i=1}^{n} \sqrt{1-C_{T i}} \\
& =\sum_{i=1}^{n}\left\{\sqrt{\left[1-4 a_{i}\left(1-a_{i}\right)\right]}\right\}=\sum_{i=1}^{n}\left(1-2 a_{i}\right), \\
& \quad n=1,2, \cdots, N,
\end{aligned}
$$

and

$$
\sum_{i=1}^{n}\left(2 a_{i}\right)=\sum_{i=1}^{n}\left(1-\sqrt{1-C_{T i}}\right), \quad n=1,2, \cdots, N .
$$

When AIF $a_{i}>\frac{1}{2}$, the momentum will break down. Meanwhile, the thrust coefficient $C_{T i}$ reaches its maximum value $\left(C_{\text {Timax }}=1\right)$ when $a_{i}=\frac{1}{2}$.

In stream tube, some of wind energy are transferred to the wind turbine when the wind flows through the wind turbine, This rotor power $P_{\text {rotori }}$ can be found theoretically by conservation of energy. We have

$$
\begin{aligned}
\sum_{i=1}^{n} \Delta P_{i} & =\sum_{i=1}^{n} P_{\text {rotori }} \\
& =\sum_{i=1}^{n}\left(\frac{1}{2} \dot{M}_{i} v_{0 i}^{2}-\frac{1}{2} \dot{M}_{i} v_{2 i}^{2}\right), \quad n=1,2, \cdots, N
\end{aligned}
$$

where $\Delta P_{i}$ is the pressure difference and $A_{r i}$ is the rotor area. Then the sum of Thrust Force $\sum_{i=1}^{n} T_{i}$ are defined as:

$$
\begin{aligned}
\sum_{i=1}^{n} T_{i} & =\sum_{i=1}^{n} \Delta P_{i} A_{r i} \\
& =\sum_{i=1}^{n} \frac{1}{2} \rho\left(v_{0 i}^{2}-v_{2 i}^{2}\right) A_{r i} \\
& =\sum_{i=1}^{n} 2 \rho v_{0 i}^{2} A_{r i} a_{i}\left(1-a_{i}\right), \quad n=1,2, \cdots, N .
\end{aligned}
$$

Meanwhile, the power of wind passing through the area where the wind turbine occupied is:

$$
\sum_{i=1}^{n} P_{\text {windi }}=\sum_{i=1}^{n}\left(\frac{1}{2} \rho v_{0 i}^{3} A_{r i}\right), \quad n=1,2, \cdots, N .
$$


The power coefficient $C_{P i}$ is defined as

$$
\begin{aligned}
\sum_{i=1}^{n} C_{P i} & =\sum_{i=1}^{n} \frac{P_{\text {RotorPoweri }}}{P_{\text {WindPoweri }}} \\
& =\sum_{i=1}^{n} \frac{P_{\text {rotori }}}{P_{\text {windi }}} \\
& =\sum_{i=1}^{n}\left(\frac{\frac{1}{2} \dot{M}_{i} v_{0 i}^{2}-\frac{1}{2} \dot{M}_{i} v_{2 i}^{2}}{\frac{1}{2} \rho v_{0 i}^{3} A_{r i}}\right), \quad n=1,2, \cdots, N .
\end{aligned}
$$

Substituting (1), (3) and (12) into the above equation (13), we have

$$
\sum_{i=1}^{n} C_{P i}=\sum_{i=1}^{n} 4 a_{i}\left(1-a_{i}\right)^{2}, \quad n=1,2, \cdots, N .
$$

According to Betz Limit Theory, the maximum value $C_{\text {Pimax }}$ will be obtained. Taking derivative of the equation (21), and make it equal to 0 , one has:

$$
\sum_{i=1}^{n} \frac{d C_{P i}}{d a_{i}}=\sum_{i=1}^{n} 4\left(1-a_{i}\right)\left(1-3 a_{i}\right)=0, \quad n=1,2, \cdots, N .
$$

Then we obtain:

$$
a_{i}=\frac{1}{3}
$$

and

$$
C_{\text {Pimax }}=4 a_{i}\left(1-a_{i}\right)^{2}=\frac{16}{27}=0.593 .
$$

Hence $C_{P i}=0.593$ reach their maximum value when $a=\frac{1}{3}$. This is so-called Betz Limit.

Further, if we solve the following equations:

$$
\left\{\begin{array}{l}
\sum_{i=1}^{n} \rho A_{r i} v_{1 i}=\sum_{i=1}^{n} \rho A_{2 i} v_{2 i}, \\
\sum_{i=1}^{n} A_{r i}=\sum_{i=1}^{n} \pi r_{r}^{2} \\
\sum_{i=1}^{n} A_{2 i}=\sum_{i=1}^{n} \pi r_{2}^{2} \\
\sum_{i=1}^{n} v_{1 i}=\sum_{i=1}^{n}\left[\left(1-a_{i}\right) v_{0 i}\right], \\
\sum_{i=1}^{n} v_{2 i}=\sum_{i=1}^{n}\left[\left(1-2 a_{i}\right) v_{0 i}\right], \quad n=1,2, \cdots, N .
\end{array}\right.
$$

We can obtain the result:

$$
\sum_{i=1}^{n} r_{2 i}=\sum_{i=1}^{n} r_{r i} \sqrt{\frac{1-a_{i}}{1-2 a_{i}}}
$$

The above equations give the main variables of the IADM.

\section{An Improved Jensen Wake Model (IJWM) for WFs}

An Improved Jensen Wake Model (IJWM) is shown in Fig. 2. In the ideal state, the sideways trapezoidal region denotes the area of the far wake. The near field followed
$W T_{1 i}$ (radius is $r_{1 i}$ ) can be treated as a turbulent wake. In the down-wind distance $x$, the circular cross-section radius is $r_{x i}$. The wind speed on $S_{1 i}$ is $v_{0 i}$, and the wind speed on $S_{x i}$ is $v_{x i}$.

The model assumes that in an ideal state, the distribution of wind speed is identical on every cross-section, and the far wake region spreads with a linear way. The trapezoidal

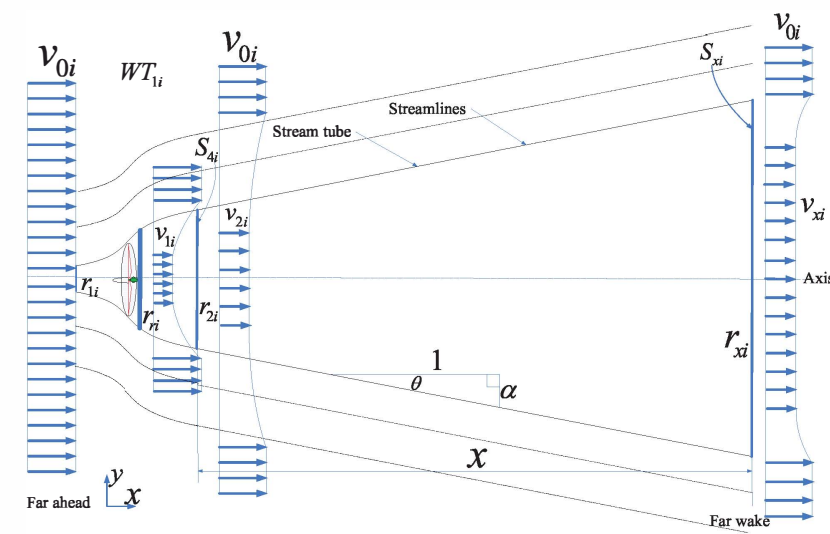

Figure 2. The portrait of Improved Jensen Wake Model (IJWM) with stream tube [11]

constitutes a tube in Fig. 2. By the law of conservation of momentum, we obtain

$$
\begin{aligned}
& \sum_{i=1}^{n} \rho \pi r_{2 i}^{2} v_{2 i}+\sum_{i=1}^{n} \rho \pi\left(r_{x i}^{2}-r_{2 i}^{2}\right) v_{0 i} \\
& =\sum_{i=1}^{n} \rho \pi r_{x i}^{2} v_{x i}, \quad n=1,2, \cdots, N .
\end{aligned}
$$

Based on the equation in the previous section,

$$
\sum_{i=1}^{n} v_{2 i}=\sum_{i=1}^{n}\left[\left(1-2 a_{i}\right) v_{0 i}\right], \quad n=1,2, \cdots, N
$$

We can joint solving equations (27), (28) and (15), and obtain the sum of velocities $\sum_{i=1}^{n} v_{x i}$, which are velocities at a distance $x$ behind the WT of $\sum_{i=1}^{n} v_{0 i}$ in the wake:

$$
\begin{aligned}
\sum_{i=1}^{n} v_{x i} & =\sum_{i=1}^{n}\left[1-2 a_{i}\left(\frac{r_{x i}}{r_{2 i}}\right)^{-2}\right] v_{0 i} \\
& =\sum_{i=1}^{n}\left[1-\frac{2 a_{i}}{\left(r_{x i} / r_{2 i}\right)^{2}}\right] v_{0 i} \\
& =\sum_{i=1}^{n}\left[1-2 a_{i}\left(1+\frac{\alpha x}{r_{2 i}}\right)^{-2}\right] v_{0 i} \\
& =\sum_{i=1}^{n}\left[1+\left(\sqrt{1-C_{T i}}-1\right)\left(\frac{r_{2 i}}{r_{x i}}\right)^{2}\right] v_{0 i} \\
& =\sum_{i=1}^{n}\left[1-\left(1-\sqrt{1-C_{T i}}\right)\left(\frac{r_{2 i}}{r_{2 i}+\alpha x}\right)^{2}\right] v_{0 i} \\
& =\sum_{i=1}^{n}\left[1-\frac{2 a_{i}}{\left(1+\alpha x / r_{2 i}\right)^{2}}\right] v_{0 i} \\
& =\sum_{i=1}^{n}\left[1-\frac{1-\sqrt{1-C_{T i}}}{\left(1+\alpha x / r_{2 i}\right)^{2}}\right] v_{0 i}, \quad n=1,2, \cdots, N .
\end{aligned}
$$


Here, $a_{i}$ is the AIF defined in the equation (2), $\sqrt{1-C_{T i}}=1-2 a_{i}, \tan \theta=\alpha=\frac{r_{x i}-r_{2 i}}{x}, r_{x i}=r_{2 i}+\alpha x$, $\sum_{i=1}^{n} v_{2 i}=\sum_{i=1}^{n}\left[\left(1-a_{i}\right) v_{0 i}\right], \sum_{i=1}^{n} v_{2 i} \stackrel{x}{=} \sum_{i=1}^{n}\left[\left(1-2 a_{i}\right) v_{0 i}\right]$, $n=1,2, \cdots, N$. The varying of wake expands with distance is defined as the scalar:

$$
\alpha=\frac{1}{2 \ln \left(\frac{h_{I i}}{h_{0 I i}}\right)}, \quad i=1,2, \cdots, N .
$$

where $h_{i}$ is the hub height of the $W T_{i}$ generating the wake, and $h_{0 i}$ is a constant called terrain roughness or surface roughness, which depends on the characteristics of the local terrain or surface.

\section{Wind Rose and Wind Weibull Probability Density Distribution}

In this section, we describe and summarize the wind rose and wind Weibull probability density distribution focusing on WFs of Luxembourg. We get the actual and real-time data from this Website [12]. Some WE cases are reduced and the WR is simplified through merging the wind speeds and wind directions in Luxembourg. These data of WE resource are collected and sorted out in the following figures. The Fig. 3 and Fig. 4 show rose portraits of average WD and WD (North=0) in Luxembourg from Jan. 01 to Mar. 31, 2015. The probabilities of most of WDs from southwest are more than $3 \%$ and approximate approaches to $4 \%$. Whereas, a small amount of WD from northeast are more than $3 \%$ and approximate approaches to $4 \%$. Therefore, WTs should face southwest in WFs of Luxembourg.

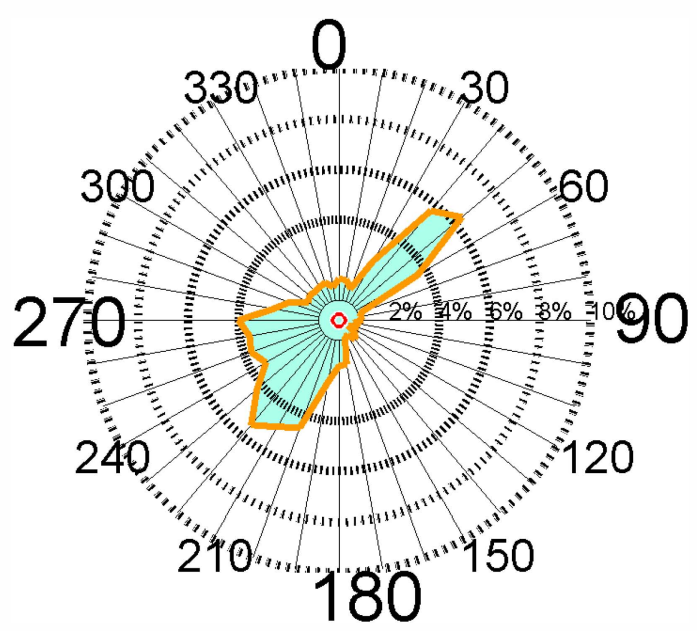

Figure 3. Rose portrait of average WD(North=0) in Luxembourg from Jan. 01 to Mar. 31, 2015.

The wind rose shows the portraits of wind mean speed $(\mathrm{m} / \mathrm{s})$ in Luxembourg from Jan. 01 to Mar. 31, 2015. The most of wind mean speeds from southwest are more than $4 \mathrm{~m} / \mathrm{s}$. Whereas, a small amount of wind mean speed from

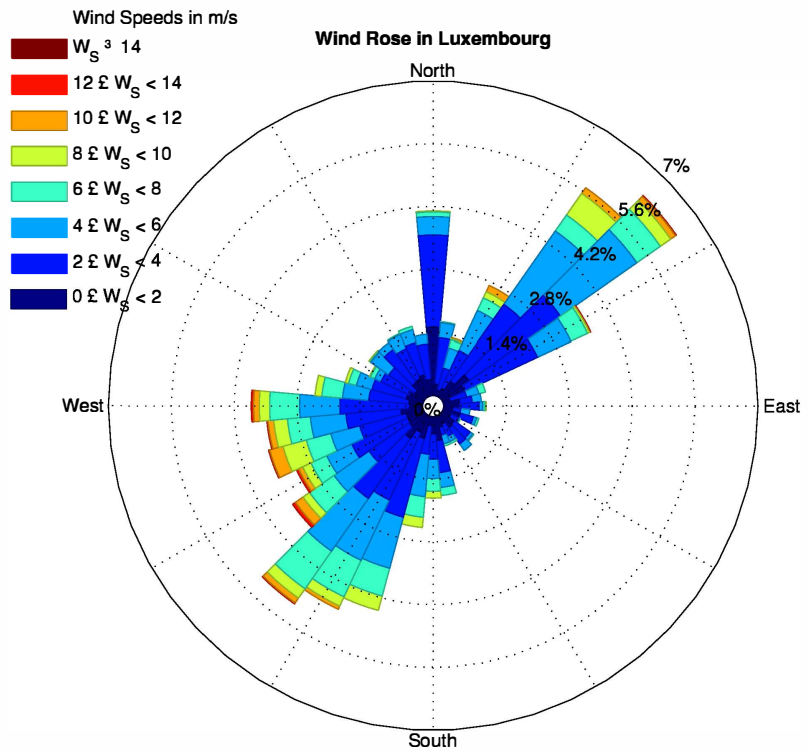

Figure 4. Rose portrait of WD(North=0) in Luxembourg from Jan. 01 to Mar. 31, 2015.

northwest are approximate to $4 \mathrm{~m} / \mathrm{s}$. Therefore, wind turbines should face to southwest in WFs of Luxembourg.

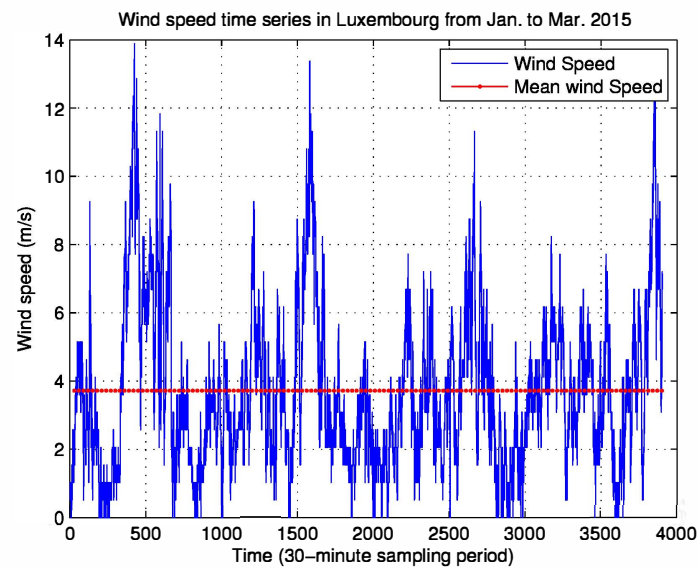

Figure 5. The portraits of wind speed and mean wind speed $(=3.7187 \mathrm{~m} / \mathrm{s})$ in Luxembourg from Jan. 01 to Mar. 31, 2015.

We continue getting the actual and real-time data from this Website [12]. The wind speed in Luxembourg are collected from Jan. 01 to Mar. 31 in 2015 and their Mean Wind Speed (MWS) is $3.7187 \mathrm{~m} / \mathrm{s}$. They are shown in the Fig. 5. Fig. 6, and Fig. 7 show the parameters and fitting curve of Weibull probability distribution for the wind speed in Luxembourg. According to wind tower, measuring data and draw wind speed histograms, Using Maximum likelihood estimation method, we estimate two parameters of the Weibull distribution, i.e. $c=4.0225$, and $k=1.6390$. Then 


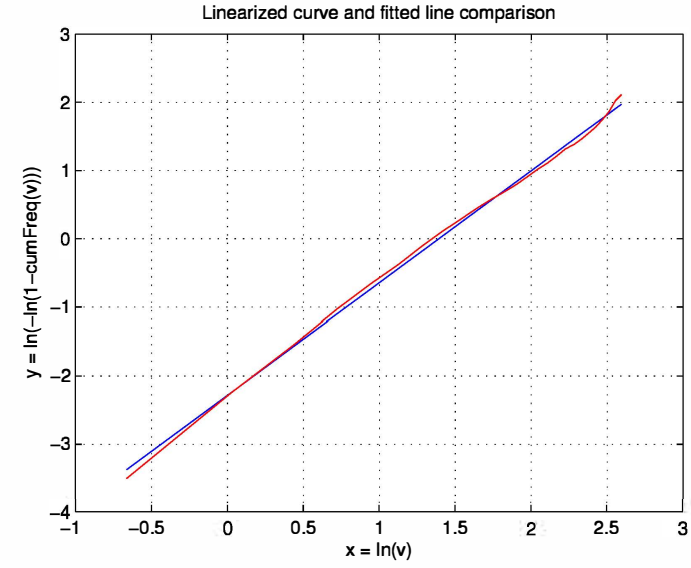

Figure 6. The portraits of linearized curve and fitted line comparison in Luxembourg from Jan. 01 to Mar. 31, 2015.
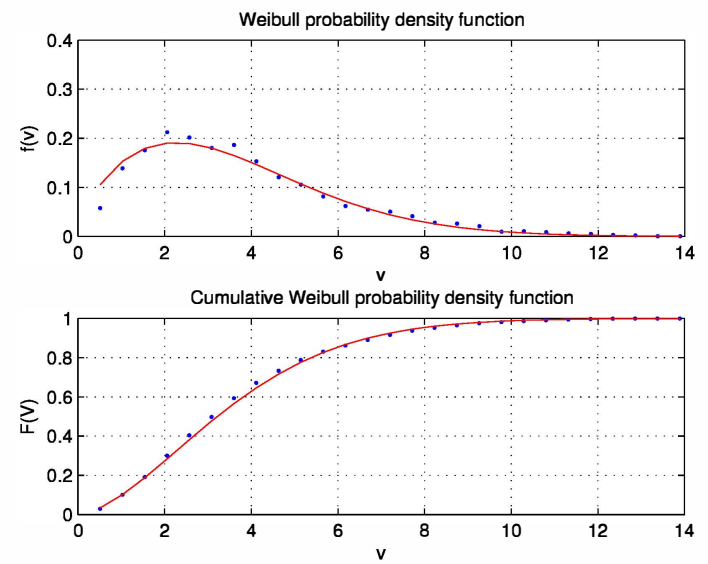

Figure 7. The portraits of functions for Weibull probability density and Cumulative Weibull probability density in Luxembourg from Jan. 01 to Mar. 31, 2015.

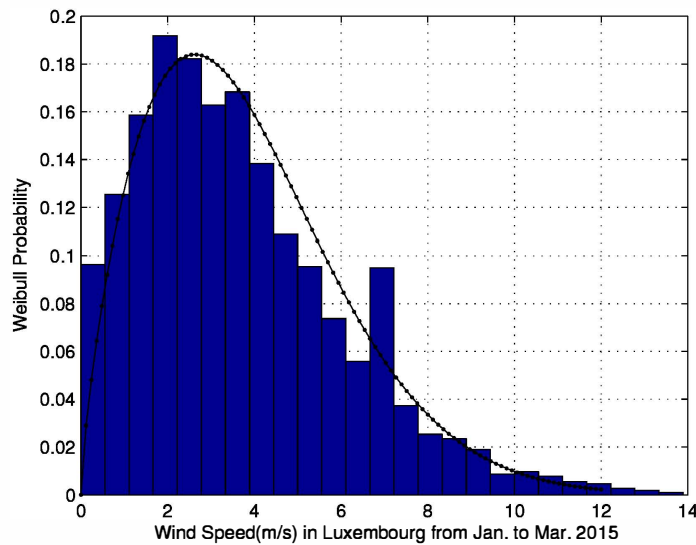

Figure 8. The portraits of wind speed histogram in hub height and the fitted Weibull probability density distribution in Luxembourg from Jan. 01 to Mar. 31, 2015. we draw the Weibull probability density distribution curve in Fig. 7. As seen in Fig. 8, the probability distribution of wind speed can be more satisfied with the Weibull distribution.

\section{Conclusions}

The paper investigates the WE systems and WFs optimization in Luxembourg. We show the overview of the wind resources in all WFs as well as present a class of IWIM for CnOC of WFs. Moreover, the statistical method is used to model the wind speed and WD distribution for WP assessment of WFs. Some simulation figures of the wind rose and Weibull distribution demonstrate the description and assessment of WP in detail. These assessments can effectively accelerate the development of WP and utilization of WFs in Luxembourg. Next step, we will study some new models of coordinated and optimization control for WFs. In addition, Large Eddy Simulation of WTs and WFs will also be the aim of our research.

\section{Acknowledgment}

This work is supported by AFR and FNR programs.

\section{References}

[1] C. Han, A. Huang, M. Baran, S. Bhattacharya, W. Litzenberger, L. Anderson, A. Johnson, and A. Edris, "Statcom impact study on the integration of a large wind farm into a weak loop power system," Energy Conversion, IEEE Transactions on, vol. 23, no. 1, pp. 226233, March 2008.

[2] J. R. Marden, S. D. Ruben, and L. Y. Pao, "A model-free approach to wind farm control using game theoretic methods," IEEE Transactions on Control Systems Technology, vol. 21, no. 4, pp. 1207-1214, 2013.

[3] K. L. Sørensen, R. Galeazzi, P. F. Odgaard, H. Niemann, and N. K. Poulsen, "Adaptive passivity based individual pitch control for wind turbines in the full load region," in Proceedings of the 2014 American Control Conference, Portland, Oregon, USA, June 4-6, 2014, pp. 554559.

[4] S. Kuenzel, L. Kunjumuhammed, B. Pal, and I. Erlich, "Impact of wakes on wind farm inertial response," Sustainable Energy, IEEE Transactions on, vol. 5, no. 1, pp. 237-245, Jan 2014.

[5] F. van Dam, P. Gebraad, and J.-W. van Wingerden, "A maximum power point tracking approach for wind farm control," Proceedings of The Science of Making Torque from Wind, 2012

[6] L. Y. Pao and K. Johnson, "A tutorial on the dynamics and control of wind turbines and wind farms," in American Control Conference, 2009. ACC '09., June 2009, pp. 2076-2089.

[7] E. Bitar and P. Seiler, "Coordinated control of a wind turbine array for power maximization," in American Control Conference (ACC), 2013, June 2013, pp. 2898-2904.

[8] L. Pan, H. Voos, Y. Li, M. Darouach, and Y. Xu, "A wake interaction model for the coordinated control of wind farms," in 20th IEEE International Conference on Emerging Technologies and Factory Automation(ETFA), September 2015, pp. 1-7, (to appear in).

[9] R. Wilson, "Wind-turbine aerodynamics," Journal of Wind Engineering and Industrial Aerodynamics, vol. 5, no. 3-4, pp. 357-372, 1980, wind Energy Conversion Systems.

[10] M. O. Hansen, Aerodynamics of wind turbines. Routledge, 2015.

[11] N. Jensen, "A note on wind generator interaction," Ris $\phi$ M 2411, November 1983.

[12] I. S. U. of Science and Technology, "The Iowa Environmental Mesonet (IEM)," http://mesonet.agron.iastate.edu/. 